\title{
Local Ensemble Kernel Learning for Object Category Recognition
}

\author{
Yen-Yu Lin ${ }^{1,2} \quad$ Tyng-Luh Liu ${ }^{1} \quad$ Chiou-Shann Fuh ${ }^{2}$ \\ ${ }^{1}$ Inst. of Information Science, Academia Sinica, Taipei 115, Taiwan \\ ${ }^{2}$ Dept. of CSIE, National Taiwan University, Taipei 106, Taiwan \\ \{yylin, liutyng\}@is.sinica.edu.tw fuhecsie.ntu.edu.tw
}

\begin{abstract}
This paper describes a local ensemble kernel learning technique to recognize/classify objects from a large number of diverse categories. Due to the possibly large intraclass feature variations, using only a single unified kernel-based classifier may not satisfactorily solve the problem. Our approach is to carry out the recognition task with adaptive ensemble kernel machines, each of which is derived from proper localization and regularization. Specifically, for each training sample, we learn a distinct ensemble kernel constructed in a way to give good classification performance for data falling within the corresponding neighborhood. We achieve this effect by aligning each ensemble kernel with a locally adapted target kernel, followed by smoothing out the discrepancies among kernels of nearby data. Our experimental results on various image databases manifest that the technique to optimize local ensemble kernels is effective and consistent for object recognition.
\end{abstract}

\section{Introduction}

Recognizing/classifying objects of multiple categories is a challenging problem. Despite much effort has been made, the performance of a state-of-the-art computer vision system is still easily humbled by human visual system, which can comfortably perform such a task with good accuracy. One major obstacle hindering the advance in developing object recognition techniques has to do with the large intraclass feature variations caused by issues such as ambiguities from clutter background, various poses, different lighting conditions, possible occlusion, etc.

Another difficulty in addressing object recognition is that its current application often deals with a large number of categories. While designing more robust visual features and their corresponding similarity measures has gained significant progress, e.g. [1, 15, 17, 19, 22], the general conclusion is that no single feature is sufficient for handling diverse objects of broad categories. Take, for example, the four object classes in Figure 1. To separate the images in jaguar
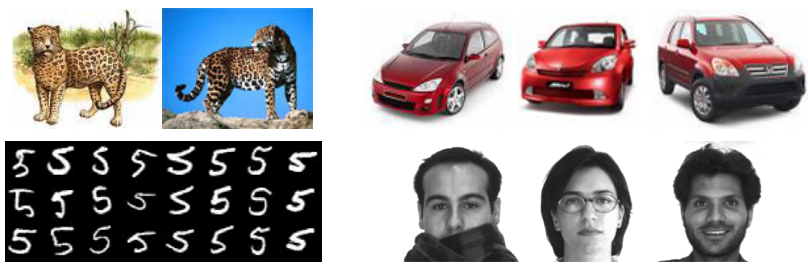

Figure 1. Examples from 4 different object categories: jaguar, red car, handwritten digit, and human face. A typical system for recognizing objects from the four categories most likely requires the use of several visual feature cues.

category from the others, keypoint-based features [15, 20] should be useful, because only those jaguar images commonly share the distinguishable patches in the skin area. On the other hand, color-based, e.g., [17] and shape-based features, e.g., [1] would be a reasonable choice for describing red car and handwritten digit, respectively. As for the human face category, it may require some proper combination of several visual features to yield a good representation. The example suggests that the goodness of a feature is often object-dependent.

Taking account of the foregoing considerations, we propose a learning approach to designing ensemble kernel machines with proper localization and regularization for object recognition. The use of ensemble kernels provides an effective way of fusing various informative kernels resulted from assorted visual features and distance functions. Consequently, it allows our recognition method to work with a large number of object categories. The framework also matches the mechanism that human visual system can receive various cues to perform recognition over diverse object classes. Even more crucially, in our formulation the learning of each ensemble kernel machine is done in a sample-dependent fashion. That is, there are as many number of local ensemble kernels as the number of training samples. In testing a new sample, a locally adapted ensemble kernel can then be efficiently constructed by referencing those from the training data. We will demonstrate that the technique can significantly alleviate the effect of intraclass variations on the outcome of object recognition. 


\subsection{Related work}

While image features for recognition can be constructed to locally or globally characterize objects of interest, their effectiveness may vary from application to application. For certain image retrieval problems, color-based histograms are conveniently adopted as a global descriptor, and shown to yield good results. For cases that they are not favorable, Pass et al. [17] propose to incorporate spatial information to form color coherence vectors (CCV). Recent trend toward resolving intraclass variations has popularized the design of local features, including region-based, part-based, and bagof-features models [8]. Local feature models can also be improved by adding spatial information [24]. Yet another possibility, as suggested in Serre et al. [19], is to devise biologically-inspired filters to output features. In a related work of Mutch and Lowe [16], the biologically-motivated filters are further consolidated for recognition.

To enhance the recognition performance, it is natural to consider combining several information cues. Berg et al. [1] introduce the geometric blur feature by investigating both the appearance and distortion evidence. In [22], Varma and Zisserman propose to merge three kinds of filter banks to generate more informative textons for texture classification. Wu et al. [23] develop super-kernel to nonlinearly combine multimodal data for image and video retrieval. It is noteworthy that in each of the aforementioned methods only a single fusion model is established for all sample points.

Besides feature fusion, the idea to learn locally adaptive classifiers has also been extensively explored. Aiming to boost nearest neighbor classification, Domeniconi and Gunopulos [7] derive a distance function for each training sample by re-weighting feature dimensions. For face recognition, Kim and Kittler [12] use $k$-means clustering to partition data, and then learn an LDA classifier for each cluster. Their method alleviates the problems caused by that data may not be linearly separable and may violate the Gaussian assumption. In [5], Dai et al. consider a responsibility mixture model, in which each sample is associated with a value of uncertainty, and use an EM algorithm to combine local classifiers based on the uncertainty distribution.

More recently, Frome et al. [10] has established a framework that for each training image, a distance function is learned by combining multiple elementary ones. Since these local distances are learned independently, testing a new image requires a more elaborate nearest neighbor search. Besides, explicitly learning as many distance functions as training data may be less efficient.

\subsection{Our approach}

We address the problem of learning sample-dependent local ensemble kernels for object recognition over diverse categories. Our method is based on energy minimization over an MRF model. Since a local learning approach sometimes tends to be subject to curse of dimensionality and the effect of noisy data, we have taken account of these issues in designing the energy function. Specifically, we employ localized kernel alignment to obtain reasonable estimates, and build from them the observation data terms in the energy function. A smoothness prior is also considered to incorporate proper regularization to the model. The proposed framework can be efficiently solved by graph cuts, and does not suffer from the out-of-sample difficulty.

\section{Information fusion via kernel alignment}

Within a kernel-based classification framework such as SVMs, the underlying kernel matrix plays the role of information bottleneck: It records the inner product of each pair of training data in some high-dimensional feature space, and has a critical bearing on the resulting decision boundary. From the Mercer kernel theory [21], any symmetric and positive semi-definite matrix is a valid kernel, in which there exists one (and only one) corresponding embedding space for the data, and vice versa.

We intend to construct a number of kernel matrices, each of them corresponds to a specific type of image feature. Combining features is thus equivalent to fusing kernel matrices. To this end, we explore the concept of kernel alignment introduced by Cristianini et al. [4]. Consider now a two-class labeled dataset $S=\left\{\left(\mathbf{x}_{i}, y_{i}\right)\right\}_{i=1}^{\ell}$ with $y_{i} \in\{+1,-1\}$. The kernel alignment between two kernel matrices $K_{1}$ and $K_{2}$ over $S$ is defined by

$$
\hat{A}\left(S, K_{1}, K_{2}\right)=\frac{\left\langle K_{1}, K_{2}\right\rangle_{F}}{\sqrt{\left\langle K_{1}, K_{1}\right\rangle_{F}\left\langle K_{2}, K_{2}\right\rangle_{F}}},
$$

where $\left\langle K_{p}, K_{q}\right\rangle_{F}=\sum_{i, j=1}^{\ell} K_{p}\left(\mathbf{x}_{i}, \mathbf{x}_{j}\right) K_{q}\left(\mathbf{x}_{i}, \mathbf{x}_{j}\right)$. With (1), the goodness of a kernel matrix $K$ with respect to $S$ can be measured by the alignment score, denoted as $\hat{A}(S, K, G)$, with a task-specific ideal kernel $G=\mathbf{y} \mathbf{y}^{T}$ where $\mathbf{y}=\left[y_{1}, \ldots, y_{\ell}\right]^{T}$.

Based on the principle of alignment to a target kernel, Lanckriet et al. [13] propose the following procedure to learn a kernel matrix for classification. First, a set of kernel matrices are generated by using different kernel functions, or by tuning different parameter values. Then, semi-definite programming (SDP) for maximizing the alignment score is performed to derive the optimal kernel as a weighted combination of the generated kernel matrices. The resulting kernel gives good performance in their experiments.

\subsection{Feature fusion over kernel matrices}

Suppose the set of training samples has $C$ classes, i.e., $S=\left\{\left(\mathbf{x}_{i}, y_{i}\right)\right\}_{i=1}^{\ell}, y_{i} \in\{1,2, \ldots, C\}$. For each $\mathbf{x}_{i} \in S$, we further assume there are totally $M$ representations arisen 
from employing various visual features. It follows that for $1 \leq r \leq M$

- The $r$ th representation of sample $\mathbf{x}_{i}$ is denoted as $\mathbf{x}_{i}^{r}$.

- $d^{r}: \mathcal{X}^{r} \times \mathcal{X}^{r} \rightarrow \mathbb{R}$ is the $r$ th distance measure, where $\mathcal{X}^{r}$ denotes the $r$ th representation domain.

Note that the representations could differ significantly. An $\mathbf{x}_{i}$ can be depicted by a histogram [22], a feature vector [12], a bag of feature vectors [15], or even a tensor. Such flexibility complicates the formulation of casting the problem of feature fusion. Several recent approaches, e.g., $[10,23,24]$ have suggested to perform information fusion in the domain of distance functions. However, a distance function can be a metric or non-metric, and the range and scale of output values by different distance functions may also vary a lot. All these issues must be well addressed to make sure if feature fusion is reasonably done.

We instead consider information fusion in the domain of kernel matrices. For each representation $r$ of the dataset $S$ and the corresponding $d^{r}$, we construct a "kernel function", similar to radial basis function (RBF) by varying the distance measure, to generate the $r$ th kernel matrix, denoted as $K^{r}$. (Note that the parameter, i.e., the variance in $\mathrm{RBF}$ function can be tuned by cross validation.) Care must be taken when $d^{r}$ is not a metric because the resulting $K^{r}$ is not guaranteed to be positive definite. Nevertheless, this issue can be resolved by the techniques suggested in [18, 24]. Let $\Omega=\left\{K^{1}, K^{2}, \ldots, K^{M}\right\}$ be the kernel bank derived by the above procedure. We define the target kernel matrix $G$ for the multiple-category object recognition problem as

$$
G(i, j)= \begin{cases}+1, & \text { if } y_{i}=y_{j} \\ -1, & \text { otherwise }\end{cases}
$$

Now multiclass feature fusion over the kernel bank $\Omega$ can be achieved by kernel alignment with respect to the target kernel $G$ defined in (2). In particular, we follow the formulation in Lanckriet et al. [13] to solve

$$
\begin{aligned}
\max _{\alpha} & \hat{A}(S, K, G) \\
\text { subject to } & K=\sum_{r=1}^{M} \alpha^{r} K^{r}, \\
& \operatorname{trace}(K)=1, \\
& \alpha^{r} \geq 0, \text { for } 1 \leq r \leq M .
\end{aligned}
$$

Alternatively, Hoi et al. [11] show that the optimization problem in (3) can be more efficiently solved using quadratic programming after reducing (3) into the follow- ing equivalent formulation:

$$
\begin{array}{cl}
\min _{\boldsymbol{\alpha}} & \boldsymbol{\alpha} D^{T} D \boldsymbol{\alpha} \\
\text { subject to } & \operatorname{vec}(G)^{T} D \boldsymbol{\alpha}=1, \\
& \alpha^{r} \geq 0, \text { for } 1 \leq r \leq M,
\end{array}
$$

where $\operatorname{vec}(A)$ is the column vectorization of matrix $A$, and $D=\left[\operatorname{vec}\left(K^{1}\right) \operatorname{vec}\left(K^{2}\right) \ldots \operatorname{vec}\left(K^{M}\right)\right]$.

Thus far we have described how to fuse $M$ visual cues through kernel alignment. An optimal $\boldsymbol{\alpha}$ of (3) or (4) uniquely determines an ensemble kernel $K=\sum_{r=1}^{M} \alpha^{r} K^{r}$. Since the derivation does not involve any local property and considers all samples, the resulting kernel $K$ is termed a global ensemble kernel. In this paper, we learn the SVM classifier by specifying $K$. And we find that in our experiments such a global ensemble kernel machine indeed achieves better recognition performance than any other SVM classifiers based on a single kernel from $\Omega$. Still, as we shall discuss in next section, the classification power can be further boosted by learning local ensemble kernels.

\section{Learning local ensemble kernels}

The previous section introduces a general principle for fusing features. Although it does provide a unified way of globally combining different feature representations, the approach is too general to account for the interclass and intraclass variations in a complex object recognition problem.

To illustrate the above point, we consider the example in Figure 2. There we have a dataset of three classes (indicated by their color), and adopt three feature representations. That is, the kernel bank $\Omega=\left\{K^{a}, K^{b}, K^{c}\right\}$. The respective feature spaces induced by $K^{a}, K^{b}$, and $K^{c}$ are (conceptually) plotted in Figures $2 \mathrm{a}-2 \mathrm{c}$. The resulting feature space by the global kernel $K_{1}=\left(K^{a}+K^{b}+K^{c}\right) / 3$, formed by a uniform combination over $\Omega$ is depicted in Figure $2 \mathrm{~d}$. While $K_{1}$ can cause a better separation among the three classes of data, it tends to misclassify samples $P$ and $Q$. Thus it appears that a local learning scheme for constructing ensemble kernels may be beneficial. In Figure 2e, the local ensemble kernel $K_{2}=\left(K^{a}+K^{b}\right) / 2$ should be effective for performing classification around $P$. Similar effect can be found for $K_{3}=\left(K^{b}+K^{c}\right) / 2$ around $Q$, as shown in Figure $2 \mathrm{f}$.

Thus, motivated by the example, and more importantly by the intrinsic difficulty in solving the recognition problem, we consider a learning formulation for constructing local classifiers. Namely, our method would derive a local ensemble kernel machine for each training sample.

\subsection{Initialization via localized kernel alignment}

In learning local ensemble kernels, intuitively one can try to generalize the idea of kernel alignment to localized kernel alignment. As it turns out, the approach would give 


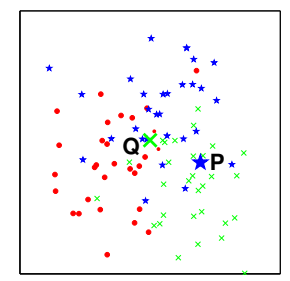

(a)

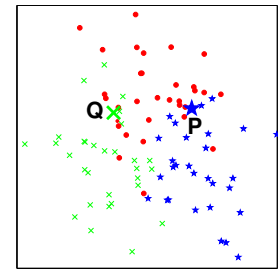

(d)

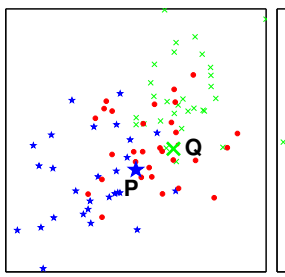

(b)

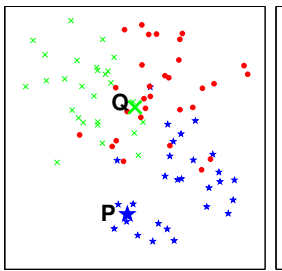

(e)

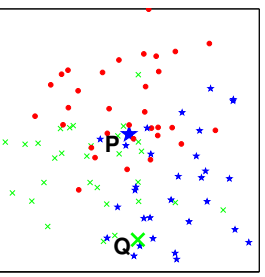

(c)
Figure 2. Six feature spaces correspond to six kernels (a) $K^{a}$, (b) $K^{b}$, (c) $K^{c}$, (d) $K_{1}=\frac{1}{3}\left(K^{a}+K^{b}+K^{c}\right)$, (e) $K_{2}=\frac{1}{2}\left(K^{a}+K^{b}\right)$, and (f) $K_{3}=\frac{1}{2}\left(K^{b}+K^{c}\right)$. See text for further details.

satisfactory results. Nevertheless we only use them as the initial observations to our proposed optimization framework for reasons that will become evident later.

We first introduce the notion of neighborhood for each sample $\mathbf{x}_{i}$. Recall that the $r$ th representation of $\mathbf{x}_{i}$ is $\mathbf{x}_{i}^{r}$, and the distance function is $d^{r}$. The neighborhood of $\mathbf{x}_{i}$ can be specified by a normalized weight vector $\mathbf{w}_{i}=$ $\left[w_{i, 1}, \ldots, w_{i, \ell}\right]$, where

$$
\begin{aligned}
& w_{i, j}=\frac{1}{M}\left(w_{i, j}^{1}+w_{i, j}^{2}+\cdots+w_{i, j}^{M}\right) \\
& w_{i, j}^{r}=\frac{\exp \left(\frac{-\left[d^{r}\left(\mathbf{x}_{i}^{r}, \mathbf{x}_{j}^{r}\right)\right]^{2}}{\sigma^{r}}\right)}{\sum_{k=1}^{\ell} \exp \left(\frac{-\left[d^{r}\left(\mathbf{x}_{i}^{r}, \mathbf{x}_{k}^{r}\right)\right]^{2}}{\sigma^{r}}\right)} .
\end{aligned}
$$

We then define the local target kernel of $\mathbf{x}_{i}$ according to $\mathbf{w}_{i}$ as follows:

$$
G_{i}(p, q)=w_{i, p} \times w_{i, q} \times G(p, q), \text { for } 1 \leq p, q \leq \ell .
$$

By replacing $G$ with $G_{i}$ in (3) and (4), we complete the formulation of localized kernel alignment. The new constrained optimization now yields an optimal vector $\boldsymbol{\alpha}_{i}$ and therefore a local ensemble kernel $K_{i}$ for each sample $\mathbf{x}_{i}$.

Note that in (7) a weight distribution is dispersed over the local target kernel $G_{i}$ such that whenever an element of $G_{i}$ relates to more relevant samples in the neighborhood of $\mathbf{x}_{i}$, it will have a larger weight (according to (5) and (6)). This property enables the resulting kernel $K_{i}=\sum \alpha_{i}^{r} K^{r}$ to be formed by emphasizing those $K^{r} \in \Omega$ that their corresponding visual cues can more appropriately describe the relations between $\mathbf{x}_{i}$ and its neighbors. Meanwhile, $\sigma^{r}$ in (6) can be used to control the extent of locality around $\mathbf{x}_{i}$. To ensure a consistent way of specifying a neighborhood for each representation $\mathbf{x}_{i}^{r}$, we adopt the following scheme:
Fixing some values of, say, $s$ and $t$, we adjust the value of $\sigma^{r}$ by binary search such that the nearest $s$ neighbors of $\mathbf{x}_{i}$, using distance $d^{r}$, will take up $t \%$ of the total weight of $\mathbf{w}_{i}$.

\subsection{Local ensemble kernel optimization}

There are two main reasons to look beyond the local ensemble kernels generated by kernel alignment. First, in most of the local learning approaches, e.g., [5, 10, 12] the resulting classifiers are determined by a relatively limited number of training samples from the neighborhood or some local group. They are sometimes sensitive to curse of dimensionality, or at the risk of overfitting caused by noisy data. To ease such unfavorable effects, we prefer a local solution with proper regularization. Second, although the kernel alignment technique in (1) has its own theoretical merit [4], we find that it does not always precisely reflect the goodness of a kernel matrix. In our empirical testing, kernel matrices that better align to the target kernel may fail to achieve better classification performance.

To address the above two issues, we construct an MRF graphical model $(V, E)$ for learning local ensemble kernels. For each sample $\mathbf{x}_{i}$, we create an observation node $\mathbf{o}_{i}$ and a state node $\boldsymbol{\beta}_{i}$. And for each pair of $\boldsymbol{o}_{i}$ and $\boldsymbol{\beta}_{i}$, an edge is connected. In addition if $\mathbf{x}_{j}$ is one of the $c$ nearest neighbors of $\mathbf{x}_{i}$ according to (5), an edge will be created between $\boldsymbol{\beta}_{i}$ and $\boldsymbol{\beta}_{j}$. Hence $|V|=2 \ell$ and $|E| \leq(c+1) \ell$. Thus there are two types of edges: $E_{1}$ includes edges connecting a state node to its observation node, and $E_{2}$ comprises those linking two state nodes. Clearly $E=E_{1} \cup E_{2}$. With the MRF so defined, we consider the following energy function:

$$
E\left(\left\{\boldsymbol{\beta}_{i}\right\}\right)=\sum_{(i, i) \in E_{1}} V_{d}\left(\boldsymbol{\beta}_{i}, \mathbf{o}_{i}\right)+\sum_{(i, j) \in E_{2}} V_{s}\left(\boldsymbol{\beta}_{i}, \boldsymbol{\beta}_{j}\right)
$$

On designing $V_{d}\left(\boldsymbol{\beta}_{i}, \mathbf{o}_{i}\right)$. In general the data term $V_{d}$ should incorporate observation evidence at $\mathbf{x}_{i}$. Specifically, we consider the neighborhood relation of $\mathbf{x}_{i}$ specified by (5) and the $\boldsymbol{\alpha}_{i}$ derived by localized kernel alignment. For the ease of algorithm design, the total number of possible states should be controlled within a manageable range. We therefore run $k$-medoids clustering (based on the alignment score) to divide $\left\{\boldsymbol{\alpha}_{i}\right\}_{i=1}^{\ell}$ into $n$ clusters, denoted as $\left\{\hat{\boldsymbol{\alpha}}_{p}\right\}_{p=1}^{n}$. ( $(n=50$ in all our experiments. $)$ The mapping $\boldsymbol{\alpha}_{i} \mapsto \hat{\boldsymbol{\alpha}}_{p(i)}$ is used to describe that $\boldsymbol{\alpha}_{i}$ is assigned to the $p(i)$ th cluster, and its vector value is approximated by $\hat{\boldsymbol{\alpha}}_{p(i)}$. Consequently, $K_{i}=\sum_{r=1}^{M} \alpha_{i}^{r} K^{r}$ is replaced by $\hat{K}_{p(i)}=\sum_{r=1}^{M} \hat{\alpha}_{p(i)}^{r} K^{r}$. In the MRF graph each observation node $\mathbf{o}_{i}$ can now be set to $\hat{\boldsymbol{\alpha}}_{p(i)}$. With these modifications, we are ready to define $V_{d}$ as follows:

$$
V_{d}\left(\boldsymbol{\beta}_{i}=\hat{\boldsymbol{\alpha}}_{q}, \mathbf{o}_{i}=\hat{\boldsymbol{\alpha}}_{p(i)}\right)=\sum_{j=1}^{\ell} w_{i, j} \times 1_{\left\{f_{q}\left(\mathbf{x}_{j}\right) \neq y_{j}\right\}}
$$


where $1_{\{\cdot\}}$ is an indicator function, and $f_{q}\left(\mathbf{x}_{j}\right)$ is the result of leave-one-out (LOO) SVM using kernel $\hat{K}_{q}$ (removing the $j$ th row and column) on $\mathbf{x}_{j}$. In practice, implementing the LOO setting in (9) is too time-consuming. Nevertheless, it can be reasonably approximated by the following scheme: Each LOO testing on $\mathbf{x}_{j}$ can be carried out by applying $f_{q}$ to $\mathbf{x}_{j}$ with the constraint that $\mathbf{x}_{j}$ cannot be one of the support vectors. (If $\mathbf{x}_{j}$ is a support vector, then remove it from $f_{q}$.)

On designing $V_{s}\left(\boldsymbol{\beta}_{i}, \boldsymbol{\beta}_{j}\right)$. We adopt the Potts model to make $V_{s}$ a smoothness prior in (8) on the free variable space. The regularization would enrich our kernel learning formulation against noisy data. Specifically, we have

$$
V_{s}\left(\boldsymbol{\beta}_{i}=\hat{\boldsymbol{\alpha}}_{q_{i}}, \boldsymbol{\beta}_{j}=\hat{\boldsymbol{\alpha}}_{q_{j}}\right)=\left\{\begin{array}{l}
0, \text { if } q_{i}=q_{j}, \\
t, \text { else if } y_{i} \neq y_{j}, \\
P \times t, \text { otherwise, }
\end{array}\right.
$$

where $t$ is a constant penalty, and constant $P \geq 1$ is used to increase penalty between state nodes $\boldsymbol{\beta}_{i}$ and $\boldsymbol{\beta}_{j}$ whose samples belong to the same object category.

With (9) and (10), the energy function in (8) is fully specified. Since finding the exact solution to minimizing (8) is NP-hard, we apply graph cuts [2] to approximating the optimal solution. Let $\left\{\boldsymbol{\beta}_{i}^{*}=\hat{\boldsymbol{\alpha}}_{p^{*}(i)}\right\}$ be the outcome derived by graph cuts. Then the optimal local ensemble kernel of $\mathbf{x}_{i}$ learned with (8) is $K_{i}^{*}=\sum_{r=1}^{M} \hat{\alpha}_{p^{*}(i)}^{r} K^{r}$. In testing, given a test sample $\mathbf{z}$, we can readily locate the nearest $\mathbf{x}_{i}$ to $\mathbf{z}$ by referencing (5), and use SVM with the local ensemble kernel $K_{i}^{*}$ to perform classification.

\section{Visual features and distance functions}

We briefly describe the image representations and their corresponding distance functions used in our experiments. These features are chosen to capture diverse characteristics of objects, such as shape, color, and texture. And our expectation is to use them to construct a kernel bank $\Omega$ that is rich enough for generating good ensemble kernels for recognizing objects of various categories. Note that hereafter a term marked in bold font is to denote a pair of an image representation and its distance measure.

\subsection{Geometric blur}

Shape-based features can provide strong evidence for object recognition, e.g., $[1,10,15]$. We adopt the geometric blur descriptor proposed by Berg et al. [1]. The descriptor summarizes the edge responses within an image patch, and is relatively robust (via employing a spatially varying kernel) to shape deformation and affine transformation. Since the optimal kernel scale can depend on several factors, e.g., the object size, we implement geometric blur descriptors with two kernel scales, and denote them as GB-L and GB-S respectively. Our implementation of geometric blur follows (2) of [24], in which spatial information is used.

\subsection{Texton}

Roughly speaking, texture feature refers to those image patterns that display homogeneity. To capture such visual cues, we consider the setting of [22], where 99 filters from three filter banks are used to generate the textons (the vocabularies of texture prototypes [14]). An image can then be represented by a histogram that records its probability distribution over all the generated textons. Like in [14, 22], the $\chi^{2}$ distance is selected as the similarity measure.

\subsection{SIFT}

We use the SIFT (Scale Invariant Feature Transform) detector [15] to find interest points in an image. To measure the distance between two images, vector quantization, as suggested in [20], is applied to transform a bag-offeatures representation to a single feature vector via clustering. Since the number of clusters can critically affect the performance, we implement two different settings: The numbers of clusters are set to 2000 (SIFT-2000) and 500 (SIFT-500) respectively. We also normalize the feature vector of each image to a distribution, and again use the $\chi^{2}$ distance as the similarity measure.

\subsection{Biologically-motivated feature}

Serre et al. [19] propose a set of features that emulates the visual system mechanism. Through a four-layer (known as $\mathrm{S} 1, \mathrm{C} 1, \mathrm{~S} 2$, and $\mathrm{C} 2$ ) hierarchical processing, an image can be expressed by a set of scale and translationinvariant $\mathbf{C} 2$ features. Motivated by their good performance for recognition, we use the $\mathrm{C} 2$ features as one of our image representations in the experiments. For this representation, Euclidean distance is applied to measuring the dissimilarity between a pair of images.

\subsection{Color related feature}

The visual features described so far catch characteristics only based on gray-level information. We thus further consider color-related image features, especially those which have compact representations and match human intuition. Specifically, we use a 125-bin color histogram $(\mathbf{C H})$ extracted from the HSV color space to represent an image. To include spatial information, the 250-bin color coherence vector (CCV) [17] is also implemented. After normalizing a $\mathrm{CH}$ or $\mathrm{CCV}$ of an image to a distribution, Jeffrey divergence is used as the distance function.

\section{Experimental results}

A real-world recognition application often involves objects from diverse and broad categories. Even for objects 


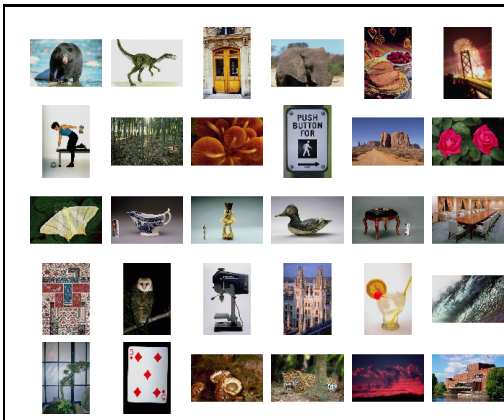

(a) Corel

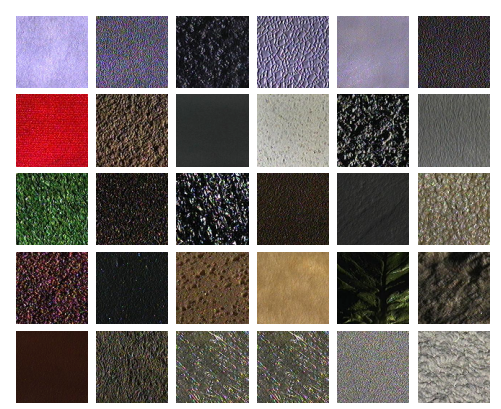

(b) CUReT

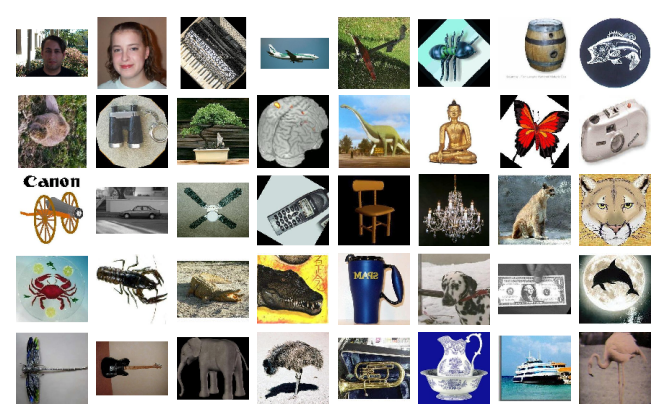

(c) Caltech-101

Figure 3. 100 image categories. (a) 30 categories from Corel, (b) 30 categories from CUReT, and (c) 40 categories from Caltech-101.

from the same category, their appearances and characteristics can still vary due to different poses, scales, or lighting conditions. Nonetheless, a problem like this serves as a good test bed for evaluating the proposed local ensemble kernel learning. In our implementation, we formulate the recognition task as a multiclass classification problem, and use LIBSVM [3], in which one-against-one rule is adopted, to learn the classifiers with the kernel matrices produced by our method. We carry out experiments on two sets of images. The first one is Caltech-101 collected by Fei-Fei et al. [9], and the second set is a mixture of images from Corel, CUReT [6] and Caltech-101. Detailed experimental results and discussions are given below.

\subsection{Caltech-101 dataset}

The Caltech-101 [9] dataset consists of 101 object categories and an additional class of background images. Each category contains about 40 to 800 images. Although objects in these images often locate in the central regions, the total number of categories (i.e., 102) and large intraclass variation still make this set a very challenging one. Some examples are shown in Figure 3c.

As the sizes of images in this set are different and some of our adopted image representations are sensitive to this issue, we resize each image into a resolution around $300 \times$ 200. (The aspect ratio is maintained.) For the sake of comparison, our experiment setup is similar to the one in Berg et al. [1] and Zhang et al. [24]. Namely, we randomly select 30 images from each category: 15 of them are used for training and the rest are used for testing. However, in [10], the class of background images (i.e., BACKGROUND_Google) is excluded from the experiments. We thus include both the two settings in our experiments, and denote them as withbackground and without-background. The quantitative results and the confusion table of testing Caltech-101 are reported in Table 1 and Figure 4a, respectively. In Table 1, the exact meanings of the abbreviations for the eight kinds of image representations, listed in the Rep. $-r$ column, have been described in Section 4. In the Method column, we
Table 1. Recognition rates for Caltech-101.

\begin{tabular}{|c|c||c|c|}
\hline \multirow{2}{*}{ Method } & \multirow{2}{*}{ Rep.-r } & \multicolumn{2}{|c|}{ Caltech-101 dataset } \\
\cline { 3 - 4 } & & With-background & Without-background \\
\hline \hline \multirow{5}{*}{$K^{r}$} & GB-L & $51.57 \%$ & $51.95 \%$ \\
\cline { 2 - 4 } & GB-S & $\mathbf{5 3 . 4 0 \%}$ & $\mathbf{5 3 . 8 6} \%$ \\
\cline { 2 - 4 } & Texton & $20.73 \%$ & $20.99 \%$ \\
\cline { 2 - 4 } & SIFT-2000 & $28.76 \%$ & $29.31 \%$ \\
\cline { 2 - 4 } & SIFT-500 & $23.86 \%$ & $24.29 \%$ \\
\cline { 2 - 4 } & C2 & $31.37 \%$ & $31.68 \%$ \\
\cline { 2 - 4 } & CH & $13.66 \%$ & $13.80 \%$ \\
\cline { 2 - 4 } & CCV & $15.10 \%$ & $15.25 \%$ \\
\hline \hline$K$ & All & $54.38 \%$ & $54.92 \%$ \\
\hline$K_{i}$ & All & $57.25 \%$ & $57.95 \%$ \\
\hline$K_{i}^{*}$ & All & $\mathbf{5 9 . 8 0} \%$ & $\mathbf{6 1 . 2 5 \%}$ \\
\hline
\end{tabular}

specify what kind of kernel matrix is used with SVM to form a kernel machine: $K^{r}$ means the kernel (in $\Omega$ ) with respect to the representation $\mathbf{x}^{r}$ is used, and analogously $K, K_{i}$ and $K_{i}^{*}$ indicate the use of an ensemble kernel derived by solving global kernel alignment (4), localized kernel alignment (7), and MRF optimization (8), respectively.

The performance gain by our technique is significant. Despite our implementation of geometric blur is less effective (the GB-L and GB-S entries in Table 1) than those reported in [24], the proposed ensemble kernel learning can still achieve state-of-the-art recognition rates.

\subsection{Corel + CUReT + Caltech-101 dataset}

In testing with Caltech-101 dataset, we observe that the performance of geometric blur descriptor [1] is noticeably dominant. This phenomenon generally causes the resulting ensemble kernel is not uniformly combined, and therefore the advantage of our method may not be fully exploited. We thus construct a second dataset by collecting images from different sources to further increase the data variations.

We first select 30 image categories from the Corel image database, which is widely used in the research of image 


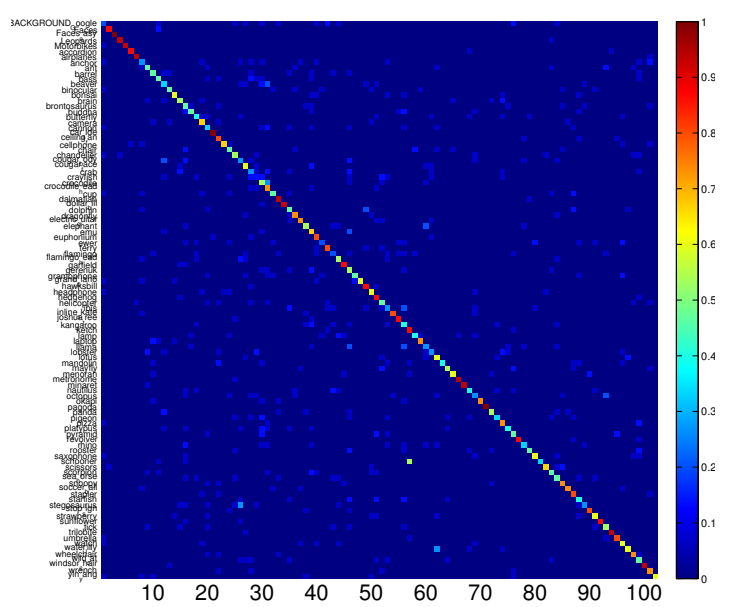

(a)

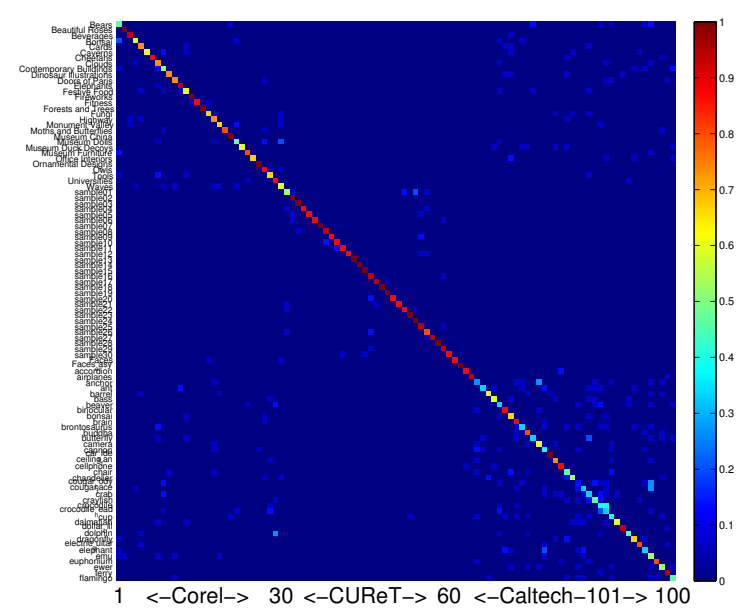

(b)

Figure 4. The confusion tables by our method on two different datasets. (a) Caltech-101. (b) Corel + CUReT + Caltech-101.

retrieval. Images in the same category share the same semantic concept, but still have their individual variety. To illustrate, images from each of the 30 categories are shown in Figure 3a. We then collect the first 30 texture categories from CUReT [6] database. Texture images within a category are pictured for the same material under various viewing angles and illumination conditions. Similar to [22], we crop the central $200 \times 200$ texture region of each image, and convert it to grayscale. However unlike in [22], we do not normalize the intensity distribution to zero mean and unit standard deviation in that this pre-processing is not applied to images from the other two sources. Figure $3 \mathrm{~b}$ gives an overview of the 30 categories selected from CUReT. Finally, we choose 40 object categories from Caltech-101 dataset according to their alphabetical order. (Note that the background category is excluded.) These categories are indeed those shown in Figure 3c. In total the resulting dataset from the three sources has 100 image categories.

Similar to the setting in Section 5.1, we randomly select 30 images from each category. Half of them are used for training, and the rest are used for testing. The quantitative results and the confusion table are shown in Table 2 and Figure $4 \mathrm{~b}$, respectively. In Table 2, there are four recognition rates recorded for each scheme. The first three are evaluated by considering only samples within specific datasets, and the last is computed by taking all samples into account.

From Table 2 and Figure 4b, we have the following observations: 1) In the Corel database, several image representations can achieve comparable performance, and tend to complement each other. Thus all the three schemes $K$, $K_{i}$ and $K_{i}^{*}$ that combine various features achieve substantial improvements. 2) Unlike testing with Caltech-101, the optimal feature combinations for classifying objects in the combined dataset are more diversely distributed. Thus the accuracy improvement of the scheme $K$ that globally learns a single fusion of visual features for all samples is relatively limited, compared with those by the two local schemes $K_{i}$ and $K_{i}^{*}$. 3) No matter using which dataset, the performance of our approach is better than the best performance obtained from using a single image representation. This means that our method can effectively select good feature combination for each sample, and thus improve the recognition rates.

Complexity analysis. Most of the time complexity in training is consumed by the construction of the kernel bank $\Omega$. It involves the pairwise distance calculations, and some of our chosen distances require extensive computation time. For 1500 training samples, this step would take several hours to complete. In addition, after kernels are grouped as clusters in the MRF optimization, learning the sampledependent SVM classifiers can be done in less than 2 minutes. In testing a novel sample, our method carries out nearest neighbor search to locate the appropriate local classifier and then performs the classification. Totally, it would take about $5 \frac{1}{2}$ minutes for testing a new sample. Finally, we remark that although a local kernel is learned for each training sample, we do not need to store the whole kernel matrix but the ensemble weights. Hence there is no extra space requirements due to our proposed method.

\section{Conclusions}

Motivated by that the best visual feature combination for classification could vary from object to object, we have introduced a sample-dependent learning method to construct ensemble kernel machines for recognizing objects over broad categories. Overall, we have strived to demonstrate such advantages with the proposed optimization framework 
Table 2. Recognition rates for Corel + CUReT + Caltech-101.

\begin{tabular}{|c|c||c|c|c|c|}
\hline \multirow{2}{*}{ Method } & \multirow{2}{*}{ Rep.-r } & \multicolumn{4}{|c|}{ Corel + CUReT + Caltech-101 dataset } \\
\cline { 3 - 6 } & & Corel (30 classes) & CUReT (30 classes) & Caltech (40 classes) & ALL (100 classes) \\
\hline \hline \multirow{3}{*}{$K^{r}$} & GB-L & $59.91 \%$ & $70.36 \%$ & $52.30 \%$ & $60.00 \%$ \\
\cline { 2 - 6 } & GB-S & $\mathbf{6 2 . 1 4} \%$ & $75.47 \%$ & $\mathbf{5 3 . 3 0} \%$ & $\mathbf{6 2 . 6 0} \%$ \\
\cline { 2 - 6 } & Texton & $53.33 \%$ & $\mathbf{8 8 . 8 9} \%$ & $23.67 \%$ & $52.13 \%$ \\
\cline { 2 - 6 } & SIFT-2000 & $45.56 \%$ & $83.56 \%$ & $30.00 \%$ & $50.73 \%$ \\
\cline { 2 - 6 } & SIFT-500 & $42.00 \%$ & $83.56 \%$ & $25.83 \%$ & $48.00 \%$ \\
\cline { 2 - 6 } & C2 & $48.22 \%$ & $59.78 \%$ & $30.00 \%$ & $34.40 \%$ \\
\cline { 2 - 6 } & CH & $55.33 \%$ & $24.22 \%$ & $16.17 \%$ & $34.33 \%$ \\
\cline { 2 - 6 } & CCV & $56.44 \%$ & $33.78 \%$ & $47.47 \%$ & $67.00 \%$ \\
\hline \hline$K$ & All & $75.78 \%$ & $86.00 \%$ & $55.83 \%$ & $73.20 \%$ \\
\hline$K_{i}$ & All & $76.67 \%$ & $\mathbf{9 2 . 8 9 \%} \%$ & $\mathbf{5 9 . 3 3 \%} \%$ & $\mathbf{7 4 . 7 3 \%}$ \\
\hline$K_{i}^{*}$ & All & $\mathbf{7 7 . 3 3 \%}$ & $92.67 \%$ & & $37 \%$ \\
\hline
\end{tabular}

over an MRF model, of which we are able to use kernel alignment to give good initializations, and also with the promising experimental results on two extensive datasets.

Acknowledgements. This work is supported in part by grants 95-2221-E-001-031 and 96-EC-17-A-02-S1-032.

\section{References}

[1] A. Berg, T. Berg, and J. Malik. Shape matching and object recognition using low distortion correspondences. In $C V P R$, pages 26-33, 2005. 1, 2, 5, 6

[2] Y. Boykov, O. Veksler, and R. Zabih. Fast approximate energy minimization via graph cuts. PAMI, 23(11):1222-1239, 2001. 5

[3] C.-C. Chang and C.-J. Lin. LIBSVM: A Library for Support Vector Machines, 2001. Software available at http://www.csie.ntu.edu.tw/ cjlin/libsvm. 6

[4] N. Cristianini, J. Shawe-Taylor, A. Elisseeff, and J. Kandola. On kernel-target alignment. In NIPS, 2001. 2, 4

[5] J. Dai, S. Yan, X. Tang, and J. Kwok. Locally adaptive classification piloted by uncertainty. In ICML, pages $225-232$, 2006. 2, 4

[6] K. Dana, B. Van-Ginneken, S. Nayar, and J. Koenderink. Reflectance and texture of real world surfaces. ACM Trans. on Graphics, 18(1):1-34, 1999. 6, 7

[7] C. Domeniconi and D. Gunopulos. Adaptive nearest neighbor classification using support vector machines. In NIPS, 2001. 2

[8] G. Dorkó and C. Schmid. Selection of scale-invariant parts for object class recognition. In ICCV, pages 634-640, 2003. 2

[9] L. Fei-Fei, R. Fergus, and P. Perona. Learning generative visual models from few training examples: An incremental bayesian approach tested on 101 object categories. In $C V P R$ Workshop on Generative-Model Based Vision, 2004. 6

[10] A. Frome, Y. Singer, and J. Malik. Image retrieval and classification using local distance functions. In NIPS, 2006. 2, $3,4,5,6$
[11] S. Hoi, M. Lyu, and E. Chang. Learning the unified kernel machines for classification. In KDD, pages 187-196, 2006. 3

[12] T.-K. Kim and J. Kittler. Locally linear discriminant analysis for multimodally distributed classes for face recognition with a single model image. PAMI, 27(3):318-327, 2005. 2, 3, 4

[13] G. Lanckriet, N. Cristianini, P. Bartlett, L. Ghaoui, and M. Jordan. Learning the kernel matrix with semi-definite programming. In $I C M L$, pages 323-330, 2002. 2, 3

[14] T. Leung and J. Malik. Representing and recognizing the visual appearance of materials using three-dimensional textons. IJCV , 43(1):29-44, 2001. 5

[15] D. Lowe. Distinctive image features from scale-invariant keypoints. IJCV, 60(2):91-110, 2004. 1, 3, 5

[16] J. Mutch and D. Lowe. Multiclass object recognition with sparse, localized features. In $C V P R$, pages 11-18, 2006. 2

[17] G. Pass, R. Zabih, and J. Miller. Comparing images using color coherence vectors. In ACM MM, pages 65-73, 1996. $1,2,5$

[18] E. Pekalska, P. Paclik, and R. Duin. A generalized kernel approach to dissimilarity-based classification. JMLR, 2(2):175-211, 2002. 3

[19] T. Serre, L. Wolf, and T. Poggio. Object recognition with features inspired by visual cortex. In $C V P R$, pages 994-1000, 2005. 1, 2, 5

[20] J. Sivic and A. Zisserman. Video google: A text retrieval approach to object matching in videos. In ICCV, pages 1470 1477, 2003. 1, 5

[21] V. Vapnik. Statistical Learning Theory. Wiley, 1998. 2

[22] M. Varma and A. Zisserman. A statistical approach to texture classification from single images. IJCV, 62(1-2):61-81, 2005. 1, 2, 3, 5, 7

[23] Y. Wu, E. Chang, K. Chang, and J. Smith. Optimal multimodal fusion for multimedia data analysis. In ACM MM, pages 572-579, 2004. 2, 3

[24] H. Zhang, A. Berg, M. Maire, and J. Malik. SVM-KNN: Discriminative nearest neighbor classification for visual category recognition. In $C V P R$, pages 2126-2136, 2006. 2, 3, 5,6 